\title{
Lentiviral siRNAs targeting multiple highly conserved $R N A$ sequences of human immunodeficiency virus type 1
}

\author{
L-J Chang, X Liu and J He \\ Gene Therapy (2005) 12, 1289. doi:10.1038/sj.gt.3302542
}

Correction to: Gene Therapy (2005) 12, 1133-1144. doi:10.1038/sj.gt.3302509

Since publication of the above article, the authors have identified an error in the Abstract. The correct abstract is shown below.

\footnotetext{
Abstract

The high mutation rate of the human immunodeficiency virus (HIV) makes it difficult for any therapy employing a single anti-HIV targeting mechanism to sustain prolonged effect. In an attempt to explore novel therapy for AIDS, we developed and tested lentiviral small interfering RNA (siRNA) vectors targeting multiple highly conserved regions in the HIV type 1 (HIV-1) genome. The siRNA expression cassette was cloned into an extensively deleted HIV-1-derived lentiviral self-inactivating (SIN) insulator vector. Although some of the siRNA targeting sites were also present in the helper construct of the vector system, the production of these lentiviral siRNA vectors was not significantly affected. When tested against different HIV-1 strains
}

including pNL4-3 (subtype B), p89.6 (subtype B), and p90CF402.1.8 (subtype A/E recombinant), the siRNAs targeting conserved gag, pol, int, and vpu, but not U3, nef, or U5 regions, efficiently inhibited replication of all three viral strains. These lentiviral siRNA vectors also protected host cells from syncytium-forming macrophage- and T-cell-tropic HIV-1-induced cytotoxicity. Transduction of a long-term chronically infected human lymphoma cell line with lentiviral siRNAs resulted in stable inhibition of HIV-1 replication. Northern analysis showed that both genomic and subgenomic viral RNA species were downregulated. In addition, the viral RNA was inhibited in both the nuclear and cytoplasmic compartments of chronically infected cells after prolonged passage, suggesting that lentiviral siRNAs have a nuclear effect. Using these lentiviral siRNA vectors, we further demonstrated reduced replication kinetics of HIV-1 in primary human peripheral blood lymphocytes. These results suggest that lentiviral siRNA vectors targeting multiple conserved HIV-1 sequences hold significant promise as a means of treating HIV-1 infection.

The authors would like to apologise for this mistake. 